\title{
Sensemaking During Unfolding Events
}

\author{
Mei-Hua Lin \\ Wright State University \\ meihua76@gmail.com
}

\author{
Helen Altman Klein \\ Wright State University \\ helen.klein@wright.edu
}

\begin{abstract}
Motivation - Organizations need to know how cognitive differences influence macrocognitive processes including information management and sensemaking. Research approach - To investigate the role of Analytic-Holistic thinking, 312 participants engaged in information management and sensemaking in the context of two unfolding scenarios. Findings/Design - Analytic-Holistic thinking directed initial sensemaking. Holistic thinkers were more influenced by information than were analytic thinkers and emphasized breadth over focus. People varied in their readiness to change their sense of a situation when faced with contradictory information. Limitations/Implications - While our scenarios were useful, more complex and possibly computer-based scenarios may better approximate naturalistic sensemaking and distributed teamwork. Originality/Value - We introduced a promising method for exploring sensemaking and information management and pioneered its use for investigating of analytic-holistic differences with implications for multinational collaborations. Take away message - In dynamic, complex domains, Analytic-Holistic thinking impacts sensemaking and information management and deserves continued attention.
\end{abstract}

\section{INTRODUCTION}

In complex domains such as commerce, military operations, and humanitarian efforts, practitioners sometimes encounter a barrage of uncertain, contradictory, and dynamic information. They must organize, interpret, and manage this information, often under time pressure, to support high stakes decision making and action. While information management provides the raw material for this sensemaking, information management also depends on sensemaking to guide the gathering and organization of incoming information. These processes help people understand, reevaluate, and act in complex situations, but they are vulnerable to variation in cognition.

When people vary in cognition, they can attend to, select, and use information differently resulting in different problems identified, interpretations constructed, and ultimately decisions made. Lin and Klein, H. A. (2008) discusses the importance of the cognitive differences proposed by Nisbett and colleagues (2001) for sensemaking in the context of cross-cultural interactions. In particular, Analytic-Holistic thinking appears important for information use and sensemaking.

This research builds on the sensemaking frameworks proposed by Weick's (1995) and Klein, G. et al. (2007). It tests the proposition that variations in analytic and holistic thinking can influence sensemaking through impacts on attention, causal attribution, tolerance for contradiction, and beliefs about change (Lin and Klein, H. A., 2008). We hypothesize that people with varying analytic and holistic tendencies will differ in the amount of information they use, the types of information they prefer, and the strategies they enlist when faced with contradictory information.

\section{METHOD}

To test our hypothesis, we used two sensemaking scenarios presenting organizational problems. Participants $(\mathrm{n}=312)$ reviewed the scenarios in which dispositional and situational information was presented sequentially. We used both quantitative and qualitative methods to explore how problem identification and judgments change as new and contradictory information was presented. Participants first made quantitative decisions and then provided qualitative responses to tap reasoning. For example, they rated the favorability of decision options and then explained what they thought had happened in the scenarios. Participants then completed the Analytic-Holism Scale (Choi, Koo, and Choi, 2007). A higher score reflects Holistic thinking while a lower score reflects Analytic thinking. Finally, a memory task assessed the participant's recall of information during sensemaking. Recalled is important because of its role in the revision and updating of judgments and plans.

\section{RESULTS}

There were four important results. First, analytic and holistic thinking influenced initial problem identification and decisions even with limited information presentation. People are not blank slates but bring with them cognitive patterns, past experiences, and beliefs that both set a framework for sensemaking, and determined how information was selected, judged, interpreted, and remembered. 
Second, analytic and holistic thinkers used information differently. Initially, holistic thinkers readily change their sensemaking based on new information and were more influenced by information type than were analytic thinkers. In our relatively brief scenarios, however, the effect of Analytic-Holistic thinking on sensemaking disappeared when new contradictory information was presented. Analytic-Holistic thinking was important for initial sensemaking, but information content dominated sensemaking in the long run.

Next, the participant's qualitative explanations for decisions showed differences in willingness to change sensemaking judgments. Some people changed readily while others were reluctant even when new contradictory information was provided. This supports the Klein, G. et al. (2007) data frame model in which contradictions or inconsistencies may motivate elaborating a frame or reconsidering previously discarded data. It also illustrated how some people were lured down a 'garden path' by explaining away the inconsistent data.

Finally, patterns of the selection and recall of dispositional and situational information varied over participants. Participant who selected and rated dispositional information to be relevant also remembered more dispositional information; those who selected and rated situational information as more relevant recalled both situational and dispositional information. Participants' selection and recall of information suggests distinctive individual information management strategies. Holistic thinkers considered the breadth of information during sensemaking while analytic thinkers focused on specific information. During teamwork, these differences can reduce the common ground needed to deal with complex problems.

\section{DISCUSSION}

The sensemaking scenarios of the type used in this study may provide a valuable test bed for probing sensemaking. They simulate the complex processes of sensemaking by varying information over time and allowing changes in decision making. They also allow the investigation how people remember and use information. Memory is an important part of retrospective sensemaking and can influence future actions. This paradigm appears useful for identifying links among information use, information recall, and sensemaking. Future efforts should use more complex scenarios so that the impact of information overload, dynamic change, and contradiction can be better explored.

As distributed teams increasingly span across national borders, variation in cognition can challenge collaborations and computer-mediated communication. When team members attend to different information, it can compromise common ground, shared sensemaking, and ultimately effectiveness. Groups from East Asia, typically more holistic in their thinking, find more information to be relevant than a U.S. group, typically more analytic in their thinking (Klein, H. A. et al., 2006). Understanding the information preferences of team members may help with information sharing by tailoring information needs during multinational teamwork (Lin et al., 2007).

We have started to identify the information management and sensemaking challenges organizations face. Like individuals, organizations need to make sense of their environment through effective information management (Weick, 1995). Organizations increasingly include people with different expertise, cognitive patterns, personalities, and cultural roots. At the same time, people in organizations face dynamic and uncertain information. Research into cognitive differences can provide guidance for information management, organizational communication patterns, sensemaking, conflict resolutions, decision making.

\section{REFERENCES}

Choi, I, Koo, M., \& Choi, J. (2007). Individual differences in analytic versus holistic thinking. Personality and Social Psychology Bulletin, 33, 691-705

Klein, H. A., Lin, M., Radford, M., Masuda, T., Choi, I., Lien, Y-W., Yeh, Y-Y., \& Chu, C. (2006). The Rosetta Project: Measuring national differences. Technical Report prepared for the Air Force Research Laboratory/Human Effectiveness, Dayton, Ohio and AOARD, Tokyo, Japan.

Klein, G., Phillips, J. K., Rall, E. L., \& Peluso, D. A. (2007). A data/frame theory of sensemaking. In R.R. Hoffman (Ed.), Expertise out of context (pp. 113-155). Mahwah, NJ: Lawrence Erlbaum Associates.

Lin, M. \& Klein, H. A. (2008). Athletes, murderers, and a Chinese farmer: Cultural perspectives on sensemaking. In J. M. C Schraagen, L. Militello, T. Ormerod, \& R. Lipshitz (Eds.), Naturalistic decision making and macrocognition (pp. 159-181). Aldershot, United Kingdom: Ashgate.

Lin, M., Klein, H. A., Radford, M. H. B, Choi, I., \& Lien, Y-W. (April, 2007). Cultural differences in information relevance: Implications for multinational teamwork. Poster presented at the 22nd Annual Conference of The Society for Industrial and Organizational Psychology, New York, NY, April 2007.

Nisbett, R. E., Choi, I., Peng, K., \& Norenzayan, A. (2001). Culture and system of thoughts: Holistic versus analytic cognition. Psychological Review, 108, 291-310.

Weick, K. E. (1995). Sensemaking in organizations. Thousand Oaks, CA: Sage Publications. 